\title{
Choix de précocité et pratiques de semis en maïsiculture irriguée du Sud-Ouest de la France : quelle diversité et comment l'expliquer?
}

\author{
Laure Maton ${ }^{1}$ \\ Delphine Leenhardt ${ }^{2}$ \\ Jacques-Eric Bergez ${ }^{2}$ \\ 1 BRGM \\ Service eau, économie \\ 1039, rue de Pinville \\ 34000 Montpellier \\ France \\ <l.maton@brgm.fr> \\ 2 Inra \\ Centre de recherches de Toulouse \\ UMR 1248 AGIR (Agrosystèmes et \\ développement territorial) \\ BP 52627 \\ 31326 Castanet Tolosan cedex \\ France \\ $<$ Delphine.Burger-Leenhardt@toulouse.inra. \\ fr> \\ $<$ Jacques-Eric.Bergez@toulouse.inra.fr>
}

\begin{abstract}
Résumé
Le choix de précocité, facteur clé de la rentabilité de la culture de maïs, est de plus en plus considéré comme un levier de gestion de l'irrigation. Estimer les précocités semées au sein d'un territoire pourrait être nécessaire pour une bonne estimation de la demande régionale en eau d'irrigation. Afin d'identifier les facteurs explicatifs de la distribution spatiale des précocités semées au sein d'un périmètre irrigué du sud-ouest de la France, nous avons analysé les pratiques recommandées et les pratiques effectives de maïsiculteurs. Il ressort une grande diversité de pratiques qui ne permet pas de se fier aux recommandations ou d'appliquer de simples règles de liaison entre dates de semis et précocité pour en connaître la distribution spatiale.
\end{abstract}

Mots clés : date de semis ; irrigation ; maïs ; précocité.

Thèmes : agronomie ; amélioration génétique ; productions végétales.

\section{Abstract \\ Cultivar earliness choice and sowing practices in irrigated maize of South- Western France. Which variability and how to explain it?}

Earliness choice is a key factor of maize profitability as well as irrigation water management. The knowledge of earliness groups in a wide area may help with correctly estimating the irrigation demand. In order to identify the explaining factors of the spatial distribution of maize earliness groups in an irrigated area of south-western France, we analysed recommended practices and actual farmer practices. We found a great diversity of practices. The estimation of their spatial distribution cannot rely only on recommended practices or simple rules linking the sowing date to an earliness group.

Key words: irrigation; maize; precocity; sowing date.

Subjects: agronomy; genetic improvement; vegetal productions. e maïs grain et semence représente $50 \%$ des surfaces irriguées en France (Buisson, 2005) et de l'eau consommée par les différentes cultures (OIE, 2007). Si les pratiques d'irrigation sont fortement responsables de cette consommation, il est important de considérer également comme des leviers potentiels de la gestion de l'irrigation : - les dates de semis, qui conditionnent le positionnement du cycle de la culture ; - le choix de précocité ${ }^{1}$ qui participe, par des différentiels de longueur de cycle et de surface foliaire, à la demande évapotranspirative de la culture.

Ainsi, à même date de semis, une variété tardive aura des besoins en eau plus élevés et plus tardifs, et un potentiel de rendement plus fort qu'une variété plus précoce (Girardin, 1999). Les stratégies

\footnotetext{
${ }^{1}$ Généralement traduite par la somme de degrés-jours (en base 6) pour deux phases du cycle de la plante: du semis à la floraison femelle et du semis à la maturité physiologique du grain.
} 
des agriculteurs influent sur la consommation en eau aussi bien à l'échelle de l'exploitation agricole qu'à l'échelle du territoire (Lorgeou et al., 2006 ; Ehrhard et al., 2005). Connaître la distribution spatiale des dates de semis et de choix de précocité sur un territoire est donc un élément clé pour gérer ou planifier la ressource en eau.

La présente étude vise à identifier et à analyser la diversité des choix de précocités et de dates de semis des maïsiculteurs d'un périmètre irrigué du sudouest de la France, leurs liens, et l'existence de facteurs explicatifs qui permettraient le géoréférencement de ces pratiques. L'étude repose sur le concept d'itinéraire technique (Sebillotte, 1978; Meynard et al., 2001) qui stipule l'existence d'une cohérence entre actes techniques à l'échelle de la parcelle. Elle s'appuie aussi sur la notion de système technique (Aubry, 2007) qui étend cette cohérence à l'échelle d'entités fonctionnelles ou de gestion de taille supérieure à la parcelle (exploitations, territoires d'action)

\section{Matériel et méthode}

\section{Zone d'étude}

L'étude a été conduite sur un périmètre irrigué de $500 \mathrm{~km}^{2}$ du sud-ouest de la France, à forte dominance monoculture de maïs irrigué et avec des enjeux sur l'eau. En années déficitaires en pluviométrie, le gestionnaire du périmètre irrigué doit arbitrer entre l'usage agricole de l'eau et le soutien d'étiage, et pour cela prévoir correctement la demande en eau d'irrigation (Trouvat, 1997). De plus, face à la récurrence de faibles étiages, les choix de systèmes de cultures sont remis en cause (FNE, 2003).

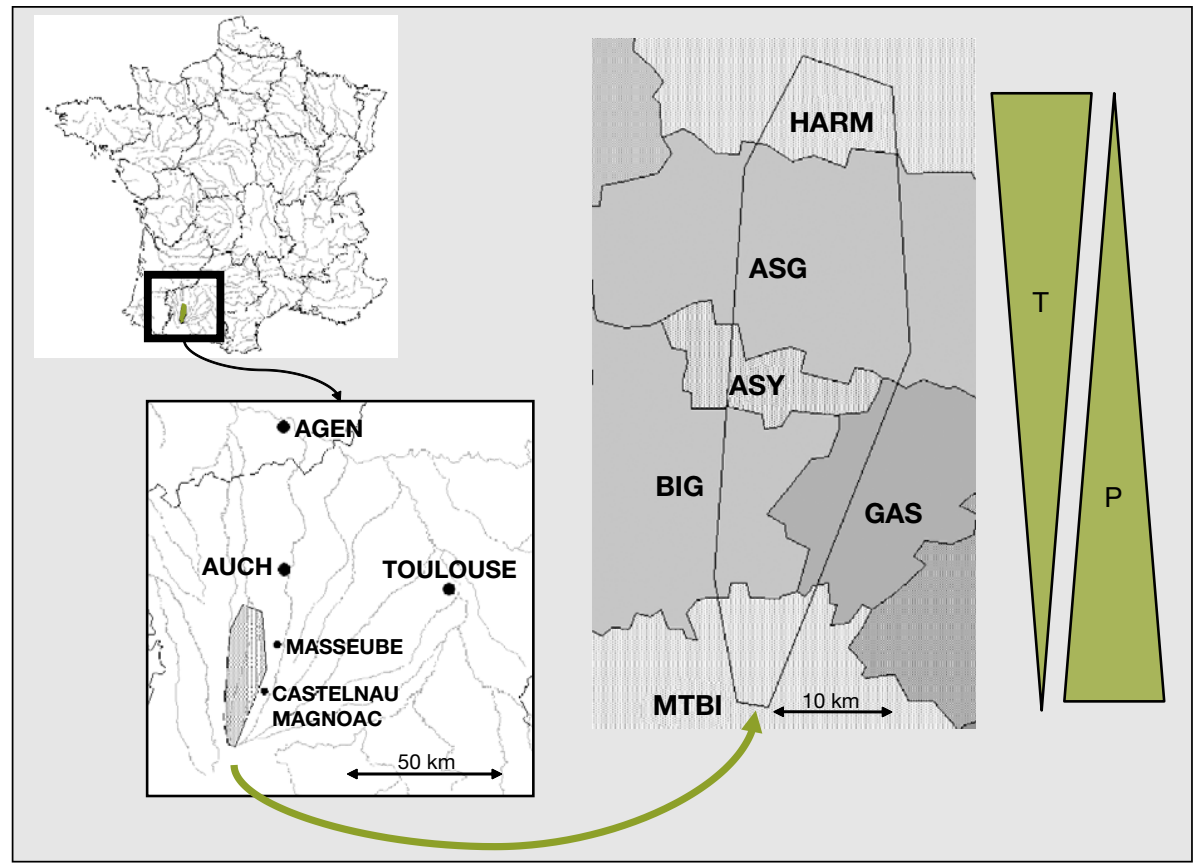

Figure 1. Localisation de la zone d'étude, et délimitation des petites régions agricoles (PRA).

Figure 1. Location of the study area and delineation of the small agricultural regions.

Haut-Armagnac (HARM), Astarac-Gers (ASG), Astarac-Hautes-Pyrénées (ASY), Coteaux-de-Gascogne (GAS), Coteaux-de-Bigorre (BIG), et Montagne-de-Bigorre (MTBI). Présentation des gradients thermiques (T) et pluviométriques $(P)$.

La zone, recoupée par cinq petites régions agricoles (PRA) (figure 1), présente un fort gradient climatique nordsud, ainsi qu'une importante variabilité interannuelle (tableau 1). Sur la durée du cycle (21 avril au 31 octobre), la médiane des sommes de température en base 6 s'étale de $2275^{\circ} \mathrm{C}$ jours au nord à $1900{ }^{\circ} \mathrm{C}$ jours au sud (Lorgeou et Souverain, 2003).

La zone est constituée de trois grands ensembles géomorphologiques: le piémont pyrénéen au sud, une alternance de larges vallées et de coteaux au centre et, au nord, des coteaux. Deux types de sol dominent. Les "terreforts", souvent sur coteaux, sont argilocalcaires. Le fort taux d'argile, qui leur confère un res- suyage lent au printemps, est le facteur limitant de l'avancement des semis, alors que leur bonne exposition de coteaux, favorable à la captation du rayonnement solaire, constitue un facteur favorable. Du fait de leur fort taux d'argile, ces sols sont labourés à l'automne. Ils nécessitent donc peu de temps de préparation juste avant le semis, d'où un temps d'intervention moindre au moment des semis. À l'opposé, les "boulbènes", plus ou moins hydromorphes selon la profondeur de la couche d'argile, et souvent en vallée, sont à dominante limoneuse. Cette propriété leur permet de se ressuyer plus vite que les terreforts, tandis que leur position basse ne leur permet pas de se réchauffer aussi vite. C'est donc le réchauffement qui

\section{Tableau 1. Pluviométrie sur la période de semis (mars à mai), pour les trois stations météorologiques proches de la zone d'étude et situées sur un axe nord-sud.}

Table 1. Rainfall during sowing period (March to May) at the three neighbouring weather stations located on a North-South axis.

\begin{tabular}{|c|c|c|c|c|c|}
\hline & $\begin{array}{l}\text { Station } \\
\text { météorologique }\end{array}$ & Moyenne (mm) & Écart type (mm) & Minimum (mm) & Maximum (mm) \\
\hline Nord & Auch & 209 & 60 & 84 & 358 \\
\hline$\downarrow$ & Masseube & 235 & 56 & 111 & 374 \\
\hline Sud & Castelnau-Magnoac & 264 & 74 & 131 & 464 \\
\hline
\end{tabular}




\section{Tableau 2. Comparaison des échantillons 2000 et 2001 avec l'ensemble de la population de maïsiculteurs irrigants de la zone d'étude.}

Table 2. Comparison of the farmers samples (2000 and 2001 postal surveys) with the total irrigated-maize growers population of the studied zone (from 2000 agricultural census data - Agreste, 2002).

\begin{tabular}{|c|c|c|c|c|c|c|}
\hline \multirow{2}{*}{$\begin{array}{l}\text { PRA } \\
\begin{array}{l}\text { Surface } \\
(\%)\end{array}\end{array}$} & \multicolumn{2}{|c|}{$\begin{array}{l}\text { Population totale } \\
\text { (500 agriculteurs) }\end{array}$} & \multicolumn{2}{|c|}{$\begin{array}{c}\text { Échantillon } 2000 \\
\text { (158 agriculteurs) }\end{array}$} & \multicolumn{2}{|c|}{$\begin{array}{l}\text { Échantillon } 2001 \\
\text { (67 agriculteurs) }\end{array}$} \\
\hline & $\begin{array}{c}\text { Agriculteurs } \\
(\%)\end{array}$ & $\begin{array}{c}\text { Surface irriguée } \\
(\%)\end{array}$ & $\begin{array}{c}\text { Agriculteurs } \\
(\%)\end{array}$ & $\begin{array}{c}\text { Surface irriguée } \\
(\%)\end{array}$ & $\begin{array}{c}\text { Agriculteurs } \\
(\%)\end{array}$ & $\begin{array}{c}\text { Surface irriguée } \\
(\%)\end{array}$ \\
\hline HARM (27) & 11 & 7 & 13 & 8 & 18 & 11 \\
\hline ASG (34) & 54 & 64 & 58,5 & 69 & 54,5 & 64 \\
\hline ASY (18) & 10 & 12 & 11,5 & 8 & 11 & 11 \\
\hline $\mathrm{BIG}(12)$ & 17 & 12 & 12,5 & 11 & 12 & 10 \\
\hline GAS (9) & 8 & 5 & 4,5 & 4 & 4,5 & 4 \\
\hline
\end{tabular}

(source: RA 2000 - Agreste, 2002). Les petites régions agricoles (PRA) échantillonnées sont: Haut-Armagnac (HARM), Astarac-Gers (ASG), AstaracHautes-Pyrénées (ASY), Coteaux-de-Gascogne (GAS) et Coteaux-de-Bigorre (BIG).

Tableau 3. Caractéristiques de différents groupes de précocité (d'après I'AGPM-Technique, 2002).

Table 3. Characteristics of the different earliness groups (adapted from AGPM-technique, 2002).

\begin{tabular}{|c|c|c|c|c|c|c|}
\hline & $\begin{array}{l}\text { Très tardif } \\
\text { (tT) }\end{array}$ & $\begin{array}{l}\text { Tardif } \\
\text { (T) }\end{array}$ & $\begin{array}{c}\text { Demi-tardif } \\
\quad(1 / 2 \mathrm{~T})\end{array}$ & $\begin{array}{c}\text { Demi-précoce } \\
(1 / 2 \mathrm{P})\end{array}$ & $\begin{array}{l}\text { Précoce } \\
\text { (P) }\end{array}$ & $\begin{array}{l}\text { Très précoce } \\
\text { (tP) }\end{array}$ \\
\hline Dénomination & E2 & E1 & D & C & B & A \\
\hline $\begin{array}{l}\text { Besoins en degrés jours } \\
\text { du semis à la récolte ( } 32 \% \\
\text { de teneur en eau du grain) }\end{array}$ & 2 200-2 005 & 2 000-1930 & $1925-1880$ & $1875-1775$ & $1775-1655$ & $1650-1555$ \\
\hline Indices de précocité & $600-700$ & $480-580$ & $390-460$ & $290-380$ & $220-280$ & $<210$ \\
\hline
\end{tabular}

est le facteur limitant de l'avancement des semis sur boulbènes. Le caractère limoneux de ces sols ne permet pas de pratiquer un labour anticipé à l'automne. Aussi, le temps d'intervention au moment du semis doit-il intégrer le labour, ce qui allonge la durée du chantier de semis.

La surface agricole utile (SAU) du périmètre est d'environ 35500 hectares. En 2000, 500 exploitations, principalement de type "polyculture élevage" et "grande culture " et représentant $32 \%$ de la SAU, ont irrigué au moins une fois et le mais grain occupait $80 \%$ des surfaces irriguées (Agreste, 2002).

\section{Méthodologie}

La méthode adoptée combine l'analyse de deux sources d'information sur les pratiques des maïsiculteurs. La première provient des organismes d'approvisionnement et de stockage (OAS) dont l'influence sur les choix de variétés des maïsiculteurs est forte (Darracq, 1996).
Une enquête a été menée, en 2003, auprès des conseillers d'OAS, couvrant la zone d'étude: trois coopératives situées dans le Gers (nord) et un négoce situé dans les Hautes-Pyrénées (sud). En plus de données quantitatives sur les semences vendues, cette enquête a permis de recueillir des informations sur les conseils apportés aux maïsiculteurs, et notamment :

- d'identifier les dates de semis et précocités recommandées aux agriculteurs ;

- de déterminer les variables permettant de décrire les pratiques de semis et de choix de précocité ;

- d'émettre des hypothèses sur les déterminants de ces pratiques et les liens entre elles.

La seconde source d'information provient des agriculteurs eux-mêmes. Deux enquêtes postales (2000 et 2001) ont porté sur les variétés de maïs semées et les dates de semis correspondantes, la surface semée à chaque date et le type de sol prépondérant sur la parcelle, et le système de production. Les retours ont été de 158 en 2000 et 67 en 2001, soit $44 \%$ ( 4735 hectares) et 18\% (1 904 hectares) des surfaces en mais grain de la zone d'étude. La distribution par PRA des agriculteurs ${ }^{2}$ et des surfaces en maïs irriguées de ces deux échantillons est conforme à celle de l'ensemble des agriculteurs de la zone (tableau 2). Les liens entre les variables décrivant le choix de précocité, les variables décrivant le chantier de semis, et les caractéristiques pédoclimatiques ont été analysés à trois échelles : la zone d'étude, la PRA et la sole de maïs grain. Le choix de précocité (tableau 3) est décrit par la proportion des différentes précocités dans la sole, ce qui informe sur le nombre de précocités utilisées et sur la précocité dominante. Le chantier de semis régit le démarrage et

\footnotetext{
$\overline{{ }^{2} \text { Chaque }}$ parcelle est affectée à la PRA du siège de l'exploitation. Cette simplification est justifiée par la faible dispersion des parcellaires d'exploitation dans la zone d'étude.
} 
l'étalement des semis de maïs dans le temps. Il est décrit par le nombre de jours de semis, la date du premier jour de semis et la durée totale du chantier qui inclut les jours sans semis entre périodes de semis. En calculant les jours disponibles pour le semis en fonction de l'état hydrique du sol (Maton et al., 2007), on peut également identifier au sein d'un chantier de semis plusieurs sessions de semis : une session correspond à l'ensemble des jours disponibles consécutifs utilisés pour semer; deux sessions sont séparées par des jours disponibles non utilisés pour le semis. La variation interannuelle des choix de précocité et du chantier de semis est analysée grâce aux 48 agriculteurs ayant répondu à l'enquête en 2000 et en 2001.

\section{Offre et conseils}

\section{en matière de précocité de maïs par les OAS}

Suite à leurs résultats commerciaux et aux résultats des essais des instituts techniques, les OAS choisissent les précocités proposées aux agriculteurs dès janvier. En 2003, les coopératives ont proposé deux précocités, tardive (T) et demitardive $(1 / 2 \mathrm{~T})$; le négoce a proposé une gamme plus large, de très tardive (tT) à demi-précoce (1/2P) (tableau 3).

Le conseil aux agriculteurs concerne le type et le nombre de précocités, et leur ordre de semis dans le temps. Qu'il soit délivré par les coopératives ou par le négoce, ce conseil est basé sur les mêmes règles générales et tient compte des sols et du climat local des parcelles, des dates de semis " habituelles " de l'agriculteur, des contraintes d'exploitation à la récolte, et des connaissances sur les rendements et résultats économiques passés. Le conseil sur les types de précocité est fonction du climat local (plus il fait chaud, plus les variétés préconisées sont tardives) et des conditions de semis. Il varie donc selon la localisation des parcelles. Ainsi, les coopératives ont l'habitude de conseiller des variétés tT et T au nord, où les semis peuvent démarrer plus tôt, tandis qu'au sud, en zone de piémont, où les semis sont plus tardifs, le négoce recommande des variétés $1 / 2 \mathrm{~T}$ et $1 / 2 \mathrm{P}$. Cependant, avec un contexte économique fluctuant et un progrès génétique le permettant, les coopératives proposent au nord de la zone de plus en plus de $1 / 2 \mathrm{~T}$. Elles permettraient :

- une sécurisation du potentiel de rendement même en cas de semis retardé;

- une baisse des coûts de séchage, car l'humidité du grain à la récolte est comparativement plus faible ;

- l'assurance que le chantier de récolte se déroule dans de bonnes conditions car, étant plus précoces, les jours disponibles pour la récolte sont plus nombreux.

Les agriculteurs ont la possibilité d'échanger les variétés commandées en cas de difficultés pour semer dans les périodes préconisées. Facile avec le négoce, cet échange est limité à $10 \%$ des commandes par les coopératives.

Pour "étaler les risques ", coopératives et négoce conseillent de semer plusieurs indices de précocité, avec un nombre de précocités proportionnel à la surface totale à semer ou à la durée totale du chantier de semis pour tenir compte de la main-d'œuvre et du matériel disponible sur l'exploitation. Ainsi, les OAS conseillent de semer une précocité donnée pour 10-20 hectares au maximum (et une variété pour 7-10 hectares) et considèrent qu'au moins deux précocités doivent être semées quand le semis est réalisé avant et après 15 mai. Toutefois, ils adaptent ce conseil à chaque exploitation en fonction de la surface à semer, des moyens techniques et humains, et des conditions pédoclimatiques. Seule la règle sur le changement de précocité après le 15 mai (Lorgeou et Souverain, 2003) est donnée comme une règle générale. Les OAS conseillent de semer d'abord les variétés les plus tardives sur les terreforts en raison de leurs caractéristiques (réchauffement rapide). Ils incitent, toutefois, les agriculteurs qui souhaitent vendre une partie de leur production au marché de soudure ${ }^{3}$ [fin août début septembre] à commencer par semer les variétés les plus précoces.

Selon les OAS, les agriculteurs ne suivent les conseils que pour 80-90\% de leur sole en maïs, le reste de la sole étant utilisé par l'agriculteur pour ses propres essais. En général, ils désirent semer des variétés plus tardives que le potentiel de température ne le permet à cause de leur fort potentiel de rendement.

\footnotetext{
$\overline{3}$ II s'agit, ici, d'une stratégie de commercialisation visant à vendre avant la production de masse, et donc à un moment où le cours du maïs est élevé.
}

\section{Pratiques des maïsiculteurs en 2000 et 2001}

\section{Sur l'ensemble de la zone}

Les dates de semis s'étalent du $1^{\mathrm{er}}$ avril au 16 juin en 2000 et du 15 avril au 15 juin en 2001. En 2000, 50 \% de la surface de maïs est semée au 8 mai, alors qu'en 2001, il faut attendre le 16 mai (figure 2).

L'ensemble des précocités tT, T et $1 / 2 \mathrm{~T}$ constitue $96 \%$ des surfaces semées en maïs en 2000 et $89 \%$ en 2001 (figure 3), mais selon des proportions variables: plus de $10 \%$ des surfaces en T en 2000 sont passées en $1 / 2 \mathrm{~T}$ en 2001. Cela peut s'expliquer par un décalage des semis d'une quinzaine de jours entre 2000 et 2001. Globalement, des variétés plus précoces sont semées quand le semis a lieu plus tard. Toutefois, pendant la période où la majorité des semis est réalisée (figure 2), tous les groupes de précocité sont semés. D'où une grande diversité de couples (date, précocité).

\section{Dans chaque petite région agricole}

La représentation des pratiques moyennes à l'échelle de la PRA révèle une cohérence globale des pratiques, entre elles et avec les conditions pédoclimatiques. Ainsi, en 2000, la zone où T est prépondérante correspond à celle où $50 \%$ des surfaces ont été semées le plus tôt (6 mai) ; lorsque le semis a été plus tardif (14 mai), 1/2T domine (figures $4 \mathrm{~A}$ et $4 B)$. En 2001, tT domine seulement sur la zone semée bien plus tôt que les autres. Cela se retrouve les deux années. Dans le Haut-Armagnac (HARM) où les semis sont plus tardifs en 2001 qu'en 2000, la précocité dominante est passée de $\mathrm{T}$ à $1 / 2 \mathrm{~T}$; dans les Coteaux-de-Gascogne (GAS) où les semis se réalisent plus tôt en 2001 qu'en 2000, la précocité dominante est passée de T à tT. En 2001, un décalage marqué (15 jours) entre le démarrage du semis des boulbènes et des terreforts peut résulter des caractéristiques différenciées des sols, mais l'ordre varie d'une PRA à l'autre, suggérant une interaction entre effets sol et climat (figure 4c).

On note, toutefois, une variabilité interannuelle des pratiques, notamment dans la distribution des précocités semées (figure 5). Ainsi, si en 2000 les distribu- 


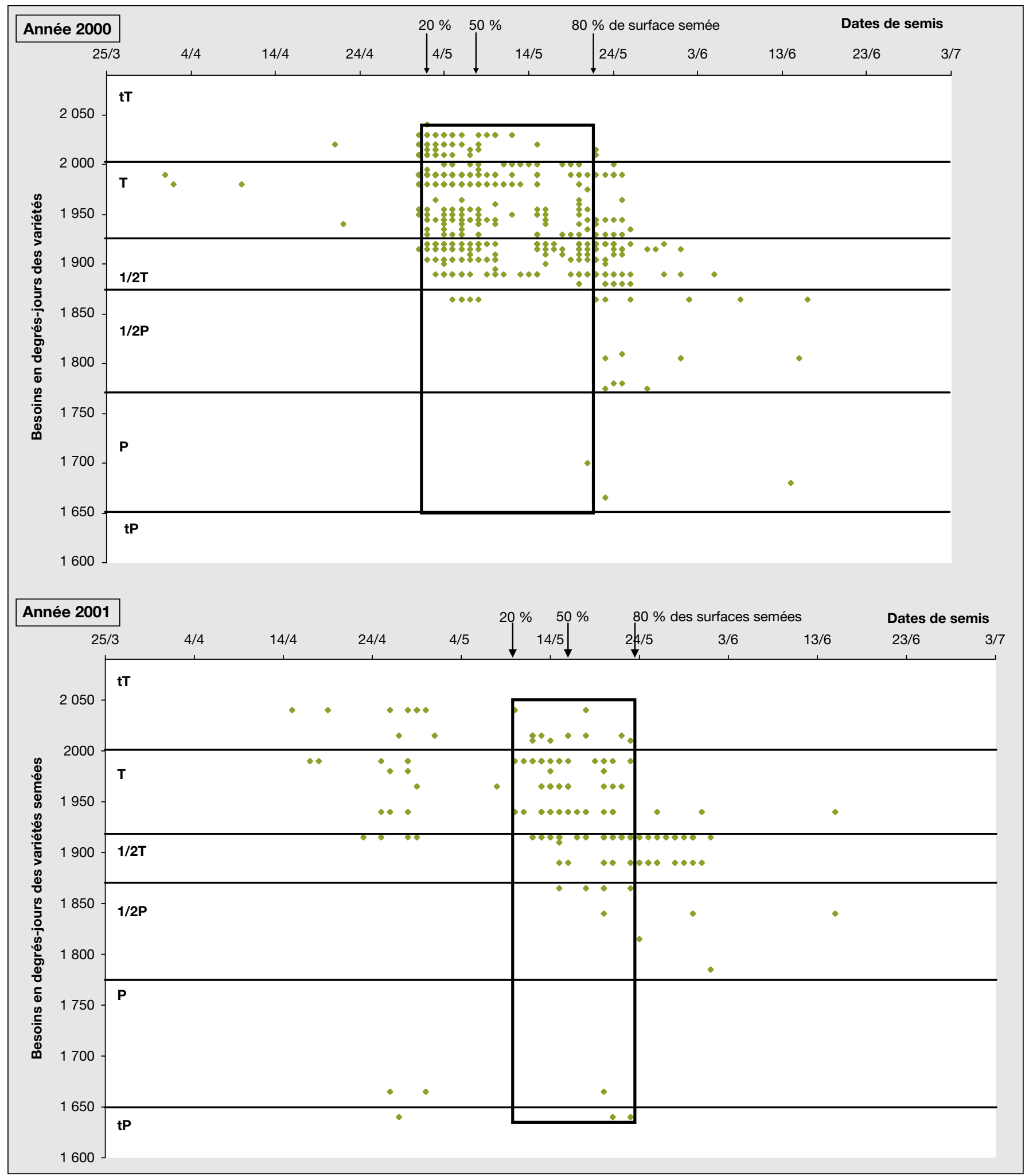

Figure 2. Dates de semis observées sur la zone d'étude, représentées en fonction des précocités semées (et de leurs besoins en sommes de température pour accomplir leur cycle du semis à la récolte $-32 \%$ de teneur en eau du grain).

Figure 2. Observed sowing dates, represented as a function of the earliness group sown (with correspondence of their heat requirements from sowing to harvest at $32 \%$ of grain humidity).

Chaque point correspond à une parcelle agricole. Les cadres regroupent les données pour la période où 20 à 80 \% des surfaces de la zone sont semées. Pour les caractéristiques des précocités : voir tableau 1. 


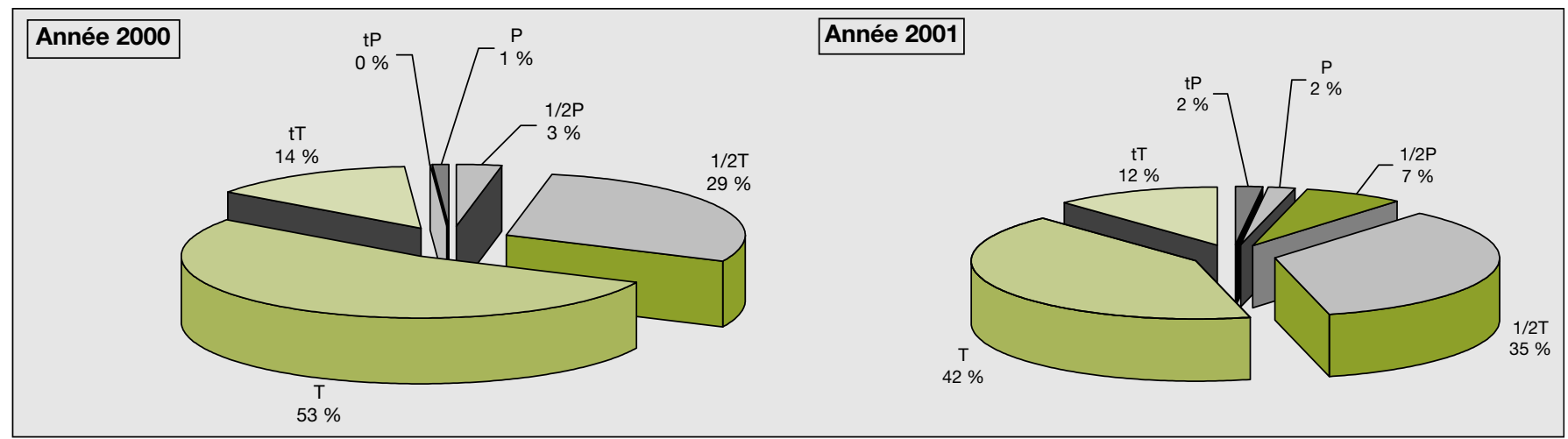

Figure 3. Part, en surface, des différentes précocités (cf. tableau 1) semées en 2000 et 2001 (données enquêtes postales).

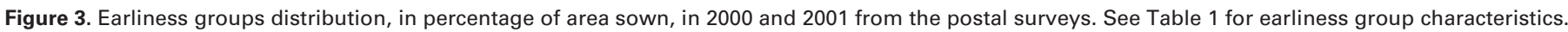
$\mathrm{T}$ : tardif ; $\mathrm{TT}$ : très tardif ; $\mathrm{P}$ : très précoce ; $\mathrm{P}:$ précoce ; $1 / 2 \mathrm{P}:$ demi-précoce ; $1 / 2 \mathrm{~T}$ : demi-tardif.

tions sont très comparables entre PRA, en 2001 elles diffèrent beaucoup du fait d'une pluviométrie plus importante au nord qu'au sud de la zone conduisant à semer plus de variétés tardives dans le sud.

\section{Au sein de la sole de maïs des agriculteurs}

Les maïsiculteurs enquêtés ont semé d'un à quatre précocités différentes (tableau 4). En 2000 et 2001, 47 et $40 \%$ d'entre eux n'ont semé qu'une seule précocité (T en 2000 et 1/2T en 2001). Parmi les autres, ceux ayant semé deux précocités prédominent. Les associations sont alors variées, mais l'association (T, 1/2T) est la plus fréquente les deux années. Parmi ceux semant trois précocités, la moitié utilise l'association (tT, T, 1/2T), tandis que l'autre moitié intègre au moins une variété de type précoce. La part des différentes précocités dans la sole varie beaucoup d'un agriculteur à l'autre, indépendamment de la surface de la sole. Les données d'enquête confirment les dires des OAS : le nombre de précocités semées croît avec la surface de la sole de maïs et avec la durée du chantier de semis. Ainsi, au-delà de 60 ha, les agriculteurs ont systématiquement semé plusieurs groupes de précocité, et des durées moyennes de chantier de semis d'une, deux, ou trois semaines correspondent respectivement à des semis d'une, deux, ou trois précocités. Il existe néanmoins une forte variabilité des surfaces et des durées de chantier pour un même nombre de précocités (tableau 4). Les données d'enquête ne permettent pas d'assurer que la présence de deux types de sol dans la sole de maïs influence le choix du nombre de précocités, même si $82 \%$ des agriculteurs ayant deux types de sol sèment plusieurs groupes de précocité contre $57 \%$ parmi ceux ayant un seul type de sol.

Les précocités semées respectent la stratification nord-sud évoquée par les OAS : au nord, les agriculteurs sont plus nombreux à privilégier des variétés tardives. En revanche, la précocité dominante dans la sole ne semble pas déterminée par la fenêtre de semis, car il n'y a pas de lien entre la date médiane du chantier de semis et la précocité dominante.

Quel que soit le type de sol, on distingue trois comportements quant à l'ordre de semis des précocités :

- les agriculteurs semant plusieurs précocités, de la plus à la moins tardive (76 et $48 \%$ en 2000 et 2001) ;

- ceux commençant par semer les variétés les plus précoces de leur sole $(6 \%$ les deux années) ;

- ceux sans ordre apparent de semis (18 et $46 \%$ en 2000 et 2001).

La première pratique, dominante et préconisée par les OAS, correspond à la recherche d'une adéquation entre potentiel de température local et besoin en chaleur des variétés. La variabilité interannuelle du nombre d'agriculteurs dans ce groupe et le transfert de certains vers le groupe 3 en 2001 questionnent sur la possibilité de suivre systématiquement cette stratégie, notamment en cas de mauvaises conditions de semis. On peut penser que les agriculteurs du groupe 2 ont une stratégie de production particulière visant l'alimentation du marché de soudure, ainsi qu'évoqué par les OAS.
L'analyse conjointe du nombre de précocités semées et de la conduite du chantier de semis permet de distinguer :

- le semis de plusieurs précocités pendant un chantier étalé (40\% des agriculteurs et $60 \%$ des surfaces en 2000);

- le semis de plusieurs précocités pendant un chantier court (26 et $19 \%$ ) ;

- le semis d'une seule précocité pendant un chantier court (28 et $18 \%$ ) ;

- le semis d'une précocité pendant un chantier étalé ( 5 et $2 \%$ ).

Un chantier étalé comprend plusieurs sessions, tandis qu'un chantier court n'en comprend qu'une seule.

L'adaptation interannuelle des agriculteurs a été étudiée en mobilisant les réponses des agriculteurs ayant répondu en 2000 et 2001. Pour $60 \%$ de ceux-ci, le nombre de précocités, les surfaces en maïs et la durée du chantier n'ont pas varié de plus de $20 \%$, en revanche, le démarrage des semis a changé, ainsi que l'ordre de semis. Pour les $40 \%$ restant, le nombre de précocités, la surface en maïs, la durée du chantier et/ ou le nombre de jours de semis a nettement varié. Pour la majorité de ces derniers, on peut supposer que les mauvaises conditions climatiques ont provoqué un retard de semis important, d'où une diminution de la surface semée par manque de temps ou de précocité adaptée, et consécutivement, une diminution du nombre de précocités. Par rapport à 2000, $25 \%$ des agriculteurs ont diminué l'indice de la précocité dominant dans la sole en 2001, 60 \% l'ont gardé identique et $15 \%$ l'ont augmenté. Le changement de précocité n'est observé qu'à partir de 15 jours de décalage de semis. Les agriculteurs, ayant diminué le nombre de précocités de deux ou trois à une, ont semé des 1/2T. 


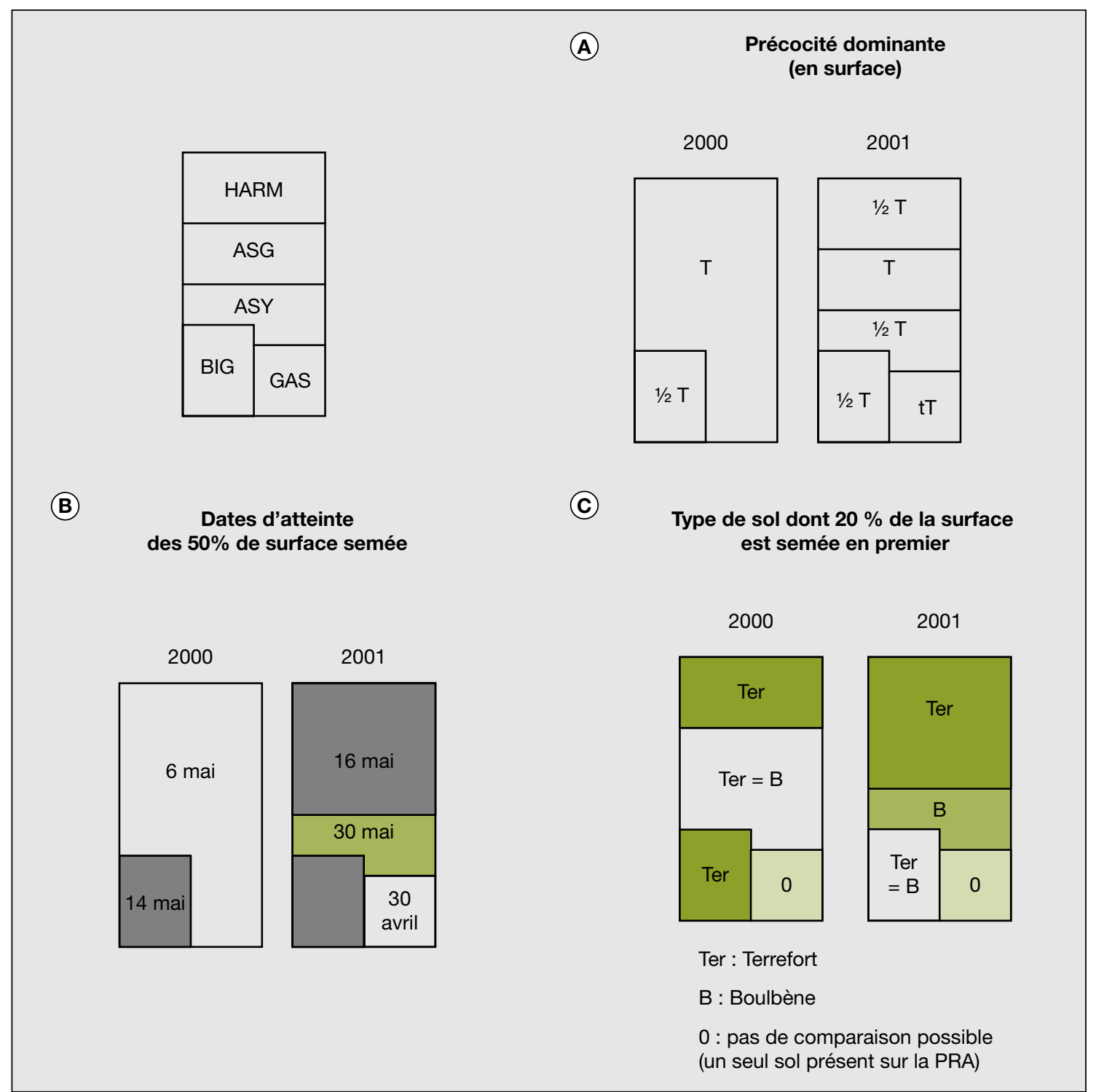

Figure 4.Zonage régional (A) des précocités dominantes ; $(B)$ des dates où $50 \%$ de la surface est semée ; (C) du type de sol semé en priorité ; et (D) des dynamiques de semis.

Figure 4. Regional zoning of (A) the majoritary earliness group; (B) the median sowing date; and (C) the soil type sown in priority.

Les petites régions agricoles (PRA) sont schématisées à gauche: Haut-Armagnac (HARM), Astarac-Gers (ASG), Astarac-Hautes-Pyrénées (ASY), Coteaux-de-Gascogne (GAS) et Coteaux-de-Bigorre (BIG).

\section{Discussion-Conclusion}

Si, au sein du territoire étudié, on retrouve bien les tendances annoncées par les conseillers, on observe une importante diversité de combinaisons (date de semis, précocité). Deux éléments peuvent expliquer cela : un gradient climatique décalant les démarrages de semis et une diversité de pratiques de choix de précocité et de semis entre maïsiculteurs à l'échelle de la sole.

L'analyse à l'échelle de la sole montre que cette diversité est liée à la variabilité des durées de chantier, au nombre de précocités semées par chaque agriculteur et à leur ordre de semis au sein de la sole. Pour ce dernier, nous avons distingué trois modes de conduite relevant de deux stratégies de production :
- semer les variétés des plus tardives au plus précoces afin d'étaler les risques climatiques ;

- semer des variétés précoces en début de période de semis pour alimenter le marché de soudure.

Les combinaisons possibles de longueur de chantier de semis et nombre de précocités semées n'ont pu être expliquées ni par la surface à semer ni par la présence 


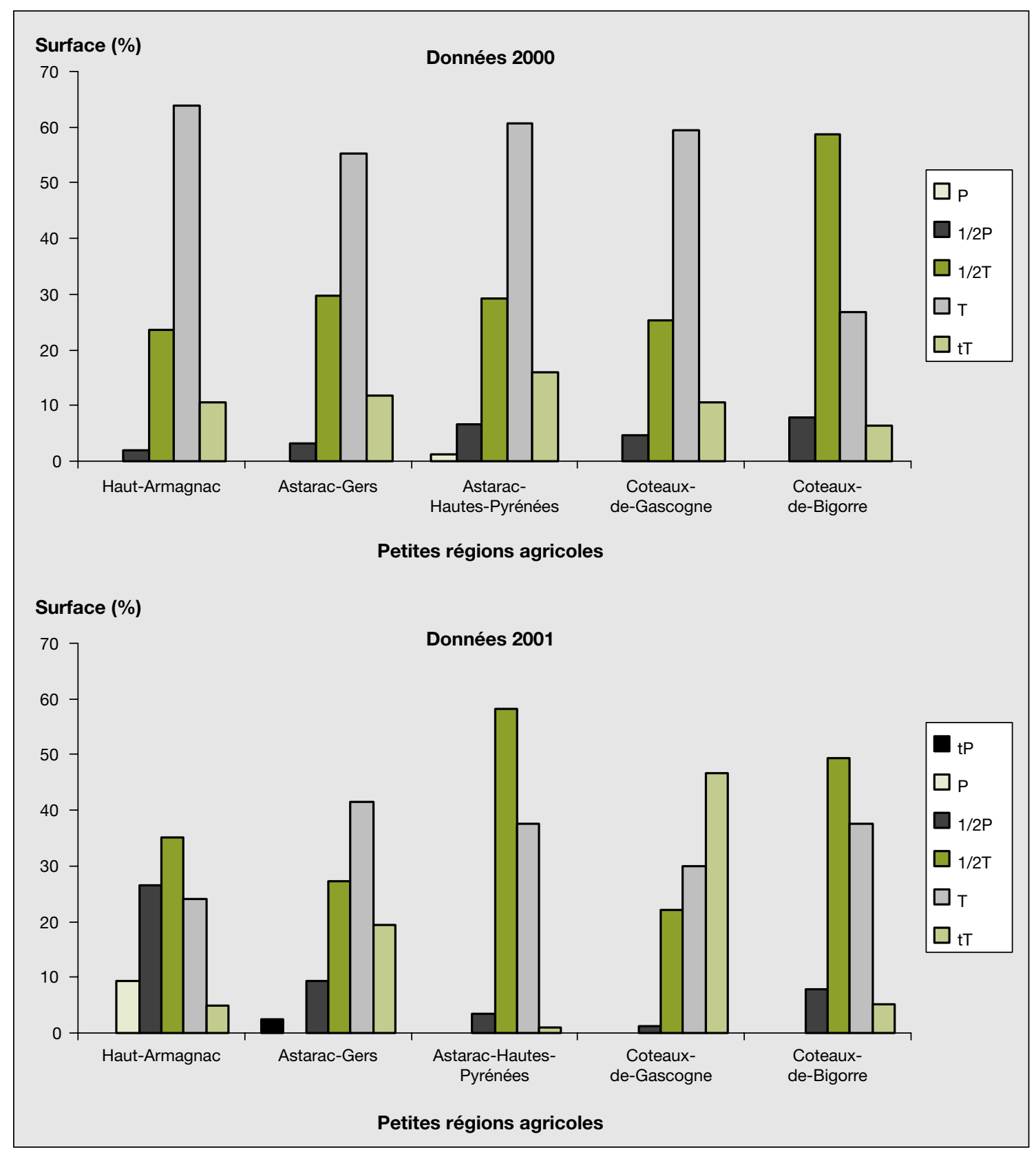

Figure 5. Précocités en pourcentage de la surface semée de chaque petite région agricole (données 2000 et 2001).

Figure 5. Earliness groups, in percentage of the area sown per small agricultural region (2000 and 2001 data). Small agricultural regions are classified from north to south.

Les petites régions agricoles (PRA) sont classées du nord au sud.

$\mathrm{T}:$ tardif ; $\mathrm{tT}$ : très tardif ; $\mathrm{P}$ : très précoce ; $\mathrm{P}$ : précoce ; $1 / 2 \mathrm{P}:$ demi-précoce ; $1 / 2 \mathrm{~T}$ : demi-tardif.

ou non de deux types de sol dans la sole. En revanche, il serait intéressant d'explorer le lien entre cette diversité de pratiques en début de saison et les stratégies de récolte des agriculteurs.
Les OAS ont finalement décrit d'une part des pratiques moyennes et d'autre part des pratiques marginales, mais bien identifiées, car correspondant à une stratégie particulière. Cette source d'information, essentiellement qualitative, ne permet pas de quantifier et de localiser les pratiques effectives. Ainsi, en terme méthodologique, l'estimation des précocités semées sur un vaste territoire ne semble 
Tableau 4. Distribution du nombre de groupes de précocité en 2000 et 2001 selon différents critères.

Table 4. Distribution of the earliness groups sown in 2000 and 2001 according to various criteria.

\begin{tabular}{|c|c|c|c|c|c|c|}
\hline \multirow[t]{2}{*}{$\begin{array}{l}\text { Nombre de groupes } \\
\text { de précocité }\end{array}$} & \multicolumn{2}{|c|}{$\begin{array}{l}\text { Nombre d'agriculteurs } \\
\text { n (\%) }\end{array}$} & \multicolumn{2}{|c|}{$\begin{array}{l}\text { Surface de maïs grain (hectares) } \\
\text { moyenne (écart type) }\end{array}$} & \multicolumn{2}{|c|}{$\begin{array}{l}\text { Durée du chantier de semis } \\
\text { (nombre de jours) } \\
\text { (moyenne et écart type) }\end{array}$} \\
\hline & 2000 & 2001 & 2000 & 2001 & 2000 & 2001 \\
\hline 1 & $73(46)$ & $27(40)$ & $17( \pm 13)$ & $20( \pm 13)$ & $3( \pm 5)$ & $5( \pm 7)$ \\
\hline 2 & $60(38)$ & $30(45)$ & $29( \pm 26)$ & $28( \pm 26)$ & $13( \pm 9)$ & $15( \pm 9)$ \\
\hline 3 & $21(13)$ & $10(15)$ & $45( \pm 43)$ & $26( \pm 43)$ & $18( \pm 8)$ & $25( \pm 10)$ \\
\hline 4 & 4 (3) & 0 & $34( \pm 18)$ & - & $21( \pm 8)$ & - \\
\hline
\end{tabular}

pouvoir se limiter à l'application de règles de liaison entre dates de semis et précocité ou à l'utilisation des prescriptions. Pour prédire les précocités semées pour des dates de semis connues ou estimées sur le territoire, il faut mettre en œuvre un modèle probabiliste qui prenne en compte la diversité des pratiques de semis et de choix de précocité des agriculteurs. Ce modèle devra simuler le nombre de précocités, leur ordre de semis et leur répartition dans la sole.

La comparaison d'informations qualitatives, recueillies auprès des distributeurs et de données d'enquêtes postales, nous a permis une identification des pratiques et de leur diversité au sein d'une grande zone géographique. Elle montre, en revanche, ses limites quant à l'analyse des raisons de cette diversité - utile pour la modélisation prospective. Avec une enquête postale simple, nous n'avons pas accès aux justifications que donneraient les agriculteurs eux-mêmes de leurs décisions techniques. Il est ainsi difficile d'évaluer ce qui relève, dans ces combinaisons de pratiques, d'aspects stratégiques et d'aspects tactiques. L'utilisation d'informations complémentaires, comme une estimation du nombre de jours disponibles pour semer, permettrait d'évaluer si la durée des chantiers de semis dépend uniquement des conditions pédoclimatiques, ou si elle peut varier suivant d'autres critères qui seraient alors propres aux contraintes d'exploitation ou aux objectifs de l'agriculteur.

\section{Remerciements}

Les auteurs remercient la Compagnie d'aménagement des Coteaux-de-Gascogne pour la diffusion des questionnaires d'enquête, les agriculteurs de la zone y ayant répondu, et les conseillers techniques ayant participé aux entretiens. Les auteurs remercient également les lecteurs anonymes de la première version de cet article pour leurs remarques constructives.

\section{Références}

AGPM-Technique. Besoins en degrés jours des variétés de maïs. Juin $2002 \mathrm{n}^{\circ} 293$.

Agreste. Recensement agricole 2000. Cartes thématiques Midi-Pyrénées. Toulouse: Service régional de la statistique agricole, 2002 (cédérom).

Aubry C. La gestion technique des exploitations agricoles. Composante de la théorie agronomique. Mémoire d'habilitation à diriger des recherches. Toulouse: INP, 2007.

Buisson G. Les effets de la réforme de la PAC de juin 2003 sur la consommation d'eau par l'agriculture. Rapport MEDD D4E. Paris: MEDD, 2005

Darracq S. Identification et modélisation des règles de décision utilisées par les maïsiculteurs en Aquitaine. Mémoire de fin d'étude INA-PG, Paris, 1996.
Ehrhard F, Escoffier I, Omnès G. L'imagination au secours du maïs irrigué. Fr Agric 2005 $3083: 61-4$.

France Nature Environnement (FNE). Charlas - Projet de barrage réservoir de soutien d'étiage. Analyse et propositions de France Nature Environnement pour une nouvelle culture de l'eau et un autre développement agricole. Paris : FNE, 2003. http://presencedutouch.free.fr/FNE/CHARLAS\%20Position\% 20FNE.pdf

Girardin P. In : Écophysiologie du maïs. Montardon: AGPM, 1999.

Lorgeou J, Souverain F. Atlas agroclimatique du maïs. Paris: Arvalis-Institut du végétal et Météo-France, 2003.

Lorgeou J, Bouthier A, Renoux JP, Cloute G. Stratégie d'évitement en maïs grain dans le Centre-Ouest : adapter le cycle aux contraintes hydriques par la précocité ? Perspect Agric $2006 ; 321: 62-8$.

Maton L, Bergez JE, Leenhardt D. Modelling the days which are agronomically suitable for sowing maize. Eur J Agron 2007 ; 27 : 123-9.

Meynard J-M, Doré T, Habib R. L'évaluation et la conception de systèmes de culture pour une agriculture durable. CR Acad Agric Fr 2001; $87: 223-36$

Office international de I'eau (OIE). Les usages agricoles de I'eau. http://www.cieau.com/toutpubl/sommaire/texte/6/contenu/613.htm.

Sebillotte $M$. Itinéraire technique et évolution de la pensée agronomique. CR Acad Agric Fr $1978 ; 64: 906-14$.

Trouvat JL. Concepts de base de la gestion quantitative de la ressource en eau. In : Lacroix $\mathrm{F}$, ed. Irrigation, outil de qualité et de régularité de la production agricole pour les marchés et les industries d'aval. Lagestion de l'eau à l'échelle d'un bassin versant: I'exemple du Sud-Ouest. Antony: AFEID-AGPM, 1997. 